\title{
Steroid cell tumor (NOS) of the ovary associated to high levels of CA-125
}

\author{
Salvatore Andrea Mastrolia • Hugo Alvarez-Arguelles • \\ Janet Carballo • Francisco Amaya • Javier de la Torre
}

Received: 31 March 2010 / Accepted: 18 May 2010/Published online: 19 June 2010

(C) Springer-Verlag 2010

\section{Introduction}

Hirsutism can be defined as an excessive male-pattern hair growth, typically caused by a state of hyperandrogenism. It can be associated with the signs of virilization, such as breast atrophy, increased muscle bulk, deepening of voice, clitoromegaly, and increased libido [1]. This report describes the case of a 44-year-old woman with a steroid cell tumor, NOS (not otherwise specified), of the ovary. This is a rare tumor of the ovary which raises differential diagnosis with many disorders causing hyperandrogenism. A very interesting element of this case is a very high level of CA-125, never described as associated to this type of tumor.

\section{Case presentation}

A 44-year-old woman presented to the University Hospital of Canarias with a 5-month history of hirsutism, amenorrhea, spotting, and choking. The patient referred a 3-year history of arterial hypertension and weight increase and a 1-year history of irregular menstrual cycle.

\section{S. A. Mastrolia}

Università degli Studi di Bari-IV Clinica Ginecologica e Ostetrica, Bari, Italy

H. Alvarez-Arguelles

Hospital Universitario de Canarias - Servicio de Anatomia Patológica, La Cuesta, Spain

S. A. Mastrolia $\cdot$ J. Carballo $\cdot$ F. Amaya $\cdot$ J. de la Torre $(\bowtie)$ Hospital Universitario de Canarias-Servicio de Ginecología y Obstetricia,

La Cuesta, Spain

e-mail: javierdelat@gmail.com
The patient had a history of interventions for leg varicose veins, hemorrhoids, bilateral tubal ligation, and two pregnancies which ended with two Cesarean deliveries.

The exploration revealed a masculinized obese female with acne and hair growth on face, arms, back, chest, and abdomen.

Laboratory analysis revealed normal values of serum cortisol, follicle-stimulating hormone, luteinizing hormone, DHEA-S, and $17 \beta$-estradiol. Serum adrenocorticotropic hormone (ACTH) value was in the range of normality, too.

A high serum value of free testosterone was found $(5.60 \mathrm{pg} / \mathrm{ml}$; normal value $<3.9 \mathrm{pg} / \mathrm{ml})$.

A SYNACTHEN ${ }^{\circledR}$ test with analogous of ACTH was performed with the results of normal values of baseline 17-OH progesterone, $30 \mathrm{~min} 17-\mathrm{OH}$ progesterone, $60 \mathrm{~min} 17-\mathrm{OH}$ progesterone, baseline insulin, baseline prolactin, and a high value of free testosterone $(9.2 \mathrm{pg} / \mathrm{ml})$.

A transvaginal ecography revealed a left adnexal mass with the diameter of $12 \mathrm{~cm}$. The mass presented two components: one solid and the second with cystic aspect, papillas, septums, and a low-resistance vascularization. Liquid free in the peritoneal cavity was found, too.

The value of CA-125 was studied with a result of 2,087.3 U/ml and a study with TAC (Fig. 1) evidenced the ovarian mass and ascitis but no peritoneal or bowel implants apparently.

The patient underwent laparotomy with intraoperatory anatomopathologic study with the result of functional steroid cell tumor. As a consequence, a total hysterectomy with bilateral salpingo-oophorectomy, omentectomy, appendicectomy, peritoneal biopsies on the bladder and paracolic tract, and iliac lymphadenectomy was performed. In the first part of the intervention, a peritoneal lavage was performed to detect the presence of neoplastic cells in the peritoneal cavity. Its analysis gave a negative result. 


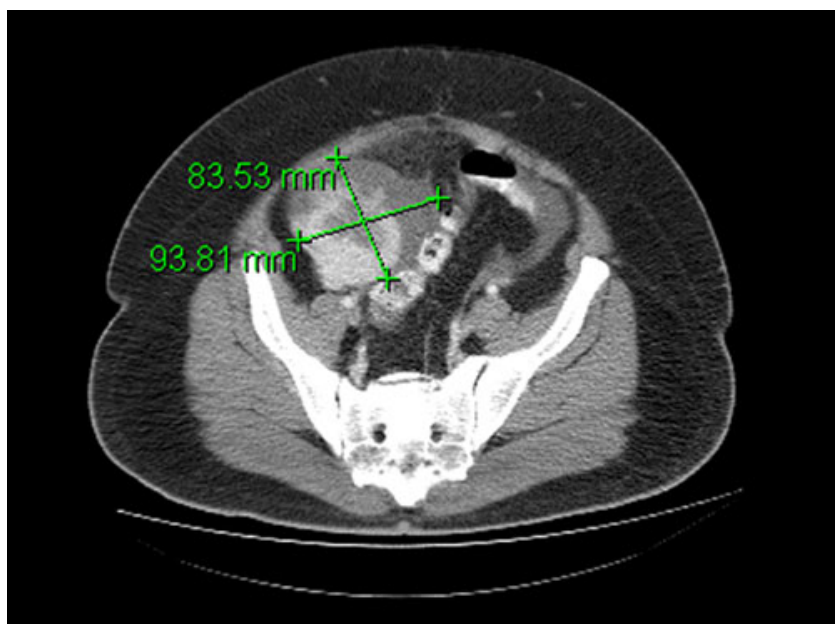

Fig. 1 Left ovarian heterogeneous mass of $93 \times 83 \mathrm{~mm}$

The macroscopic findings on the tumor were of an ovarian tumor with a diameter of $6.7 \mathrm{~cm}$ with a white surface, containing a solid lobulated yellow mass measuring $6.7 \times$ $3.5 \mathrm{~cm}$. There was no significant necrosis and hemorrhage.

The histological section revealed sheets and cords of polygonal neoplastic cells with small round nuclei, an abundant amount of vacuolated eosinophilic cytoplasm, mild atypia, and no mitosis. No Reinke's crystals were present. Fat stains were positive. These findings were consistent with a benign ovarian steroid cell tumor, NOS.

Immunohistochemistry demonstrated a focal positivity to cytokeratin and vimentin and a high positivity for inhibin alpha. The study for actin, desmin, CD68, S100 protein, and epithelial antigen of membrane was negative. After the surgery, a clinical control every 6 months was recommended to the patient. The laboratory analysis evidenced normalized hormonal levels.

The signs and symptoms have disappeared and the value of CA-125 2 years after the treatment is normal $(15.1 \mathrm{U} / \mathrm{ml})$. After this period of time, the patient remains alive and free of disease after a period of 3 years.

\section{Discussion}

Steroid cell tumors were first termed by Scully in 1979 which account for $<0.1 \%$ of all ovarian tumors [2]. They are classified among the other types of sex cord stromal tumors (the others are granulosa-stromal cell tumors, Sertoli-stromal cell tumors).

These tumors are typically solid and well circumscribed, are occasionally lobulated, and have a mean diameter of $8.4 \mathrm{~cm}$. The section surface is typically yellow or orange if large amounts of intracytoplasmatic lipid are present, red to brown if the cells are lipid-poor, or dark brown to black if large quantities of intracytoplasmatic lypocrome pigment are present. Necrosis, hemorrhage, and cystic degeneration are occasionally observed. In the $60 \%$ of the cases, nuclear atypia is absent or minimal, and mitotic figures are fewer than two per ten high-power fields. Immunohistochemical staining is positive for inhibin alpha, vimentin (75\%), CAM 5.2 (46\%), AE1/AE3, CK1 (37\%), epithelial membrane antigen (8\%), and S-100 protein (7\%) [3].

There are three steroid cell tumor subtypes: steroid cell tumors, NOS, stromal luteomas, and Leydig cell tumors. Steroid cell tumors, NOS, account for approximately $60 \%$ of steroid cell tumors. The steroid cell tumors, NOS, usually occur in younger individuals (mean age, 43 years) and, in contrast to other steroid cell tumors, occasionally occur before puberty. Hirsutism and virilization are the most common symptoms occurring in $56-77 \%$ of patients [4], because of the production of androgens.

It is not unusual for steroid cell tumors, NOS, to produce hormones other than testosterone. Estradiol secretion by these tumors is not uncommon, occurring in $6-23 \%$ of patients. Estrogen production can result in menorrhagia and postmenopausal bleeding. It was not the case of our patient. Steroid cell tumors, NOS, have also been associated with Cushing's syndrome in $6-10 \%$ of cases. Approximately $25 \%$ of steroid cell tumors, NOS, do not produce hormones. The proportion of tumors that are malignant has ranged from $25 \%$ to $43 \%$ [5].

In Hayes and Scully's work about the steroid cell tumors, NOS, the following pathologic malignancy features are indicated: two or more mitotic figures per ten high-power fields related with $92 \%$ malignancy, necrosis with $86 \%$ malignancy, a diameter of $>7 \mathrm{~cm}$ with $78 \%$ malignancy, hemorrhage associated with $77 \%$ malignancy, and grade 2 or 3 nuclear atypia with $64 \%$ malignancy [6].

In the case of our patient, histological criteria of malignancy did not exist in the intraoperative study, but an ultrasound diameter of more than $7 \mathrm{~cm}$ existed, with complex ultrasound features. For these reasons, the surgeons decided to be as radical as possible yet avoiding to be excessively aggressive. So, they did not complete the staging with para-aortic lymphadenectomy.

In every case of hyperandrogenism and its manifestations, it is very important to find the source of the hormone production.

We have to differentiate among polycystic ovary syndrome, congenital adrenal hyperplasia, hyperprolactinemia, Cushing's disease, hyperinsulinemia, or tumors producing androgens (adrenal or ovarian).

In our case, laboratory analysis detected increased levels of free testosterone as confirmed with a test using an analogous of ACTH. This also excluded the congenital adrenal hyperplasia. 
We can say that, in virilized patients, a high level of serum testosterone with no 21-hydroxylase deficiency can be considered as evidence of the presence of ovarian virilizing tumor. Otherwise, serum value of testosterone is a good tumor marker for the follow-up of the disease.

As a demonstration, the levels of testosterone became normal after the treatment and the patient lives free of disease with normal androgens blood levels.

Anyway, imaging is very useful to the purpose of a diagnostic evaluation of a woman with hirsutism and virilization. In our case, the ultrasound imaging demonstrated the presence of an adnexal mass, and a TAC excluded any pathologic process in the adrenal gland, clarifying the ovarian etiology.

Another clue derived from the value of CA-125 $(2,087.3 \mathrm{U} / \mathrm{ml})$.

CA-125 is the most well-characterized biomarker for ovarian cancer [7]. Serum concentrations of CA-125 are elevated (CA-125 levels of less than $35 \mathrm{U} / \mathrm{ml}$ are accepted as normal [8]) in more than $90 \%$ of patients with a late-stage disease but are elevated in only $50 \%$ of patients with stage I disease. CA-125 is expressed by fetalamniotic and coelomic epithelium and in adult tissues derived from the coelomic (mesothelial cells of the pleura, pericardium, and peritoneum) and Mullerian (tubal, endometrial, and endocervical) epithelia. The surface epithelium of normal ovaries does not express CA-125 [9].

In addition, elevated levels of CA-125 are strongly associated with serous tumors than mucinous tumors, but a high level of the marker has never been associated with a steroid cell tumor, NOS, of the ovary.

The treatment of this kind of tumor should be the surgery. In this case, the patient underwent total abdominal hysterectomy with bilateral salpingo-oophorectomy and complete surgical staging because of the size of the tumor. Anyway, in the absence of criteria of malignancy, a conservative surgery with unilateral oophorectomy with proper surgical staging should be performed.

Adjuvant chemotherapy should be based on the histological appearance of the tumor and on its surgical stage [10].

\section{Conclusion}

A careful history, physical examination, serum value of testosterone, and other hormones for differential diagnosis with other diseases producing similar signs and symptoms, and ultrasound imaging studies are really helpful to the diagnostic evaluation in women with hirsutism and virilization. A high level of CA-125 can be suggestive of the ovarian localization of the tumor and can help to complete the diagnostic process.

Declaration of interest The authors report no conflicts of interest. The authors alone are responsible for the content and writing of the paper.

\section{References}

1. Ehrmann DA (2008) Hirsutism and virilization. In: Fauci AS, Kasper DL, Longo DL, Braunwald E, Hauser SL, Jameson LJ, Loscalzo J (eds) Harrison's principles of internal medicine, 17th edn. McGraw-Hill, New York, pp 301-304

2. Andrade LALA, Gentilli ALC, Grimalde P (2001) Case report: sclerosing stromal tumor in an accessory ovary. Gynecol Oncol 81:318-319

3. Scully RE, Young RH, Path FRC et al (1998) Steroid cell tumors. In: Rosai J, Sobin LH (eds) Tumors of the ovary, maldeveloped gonads, Fallopian tube, and broad ligament. AFIP, Washington, pp 232-237

4. Wang PH, Chao HT, Lee RC et al (1998) Steroid cell tumor of the ovary: clinical, ultrasonic, and MRI diagnosis: case report. Eur J Radiol 26:269-273

5. Wang PH, Chao HT, Lee WL (1998) Use of a long-acting gonadotropin-releasing hormone agonist for treatment of steroid cell tumors of the ovary. Fertil Steril 69:353-355

6. Hayes MC, Scully RE (1987) Ovarian steroid cell tumor (not otherwise specified). A clinicopathological analysis of 63 cases. Am J Surg Pathol 11:835-845

7. Nossov V, Amneus M, Su F et al (2008) The early detection of ovarian cancer: from traditional methods to proteomics. Can we really do better than serum CA-125? Am J Obstet Gynecol 199(3):215-223

8. Bast RC Jr, Klug TL, St John E et al (1983) A radioimmunoassay using a monoclonal anti-body to monitor the course of epithelial ovarian cancer. N Engl J Med 309:883-887

9. Kabawat SE, Bast RC Jr, Bhan AK et al (1983) Tissue distribution of a coelomic-epithelium-related antigen recognized by the monoclonal antibody OC125. Int J Gynecol Pathol 2:275-285

10. Ready MB, Richards WE, Ueland F et al (1999) Ovarian steroid cell tumors, not otherwise specified: a case report and literature review. Gynecol Oncol 75:293-297 